\title{
Approximation by limit q-Bernstein operator
}

\author{
Zoltán Finta \\ Babeş-Bolyai University \\ Department of Mathematics \\ 1, M. Kogălniceanu st., 400084 \\ Cluj-Napoca, Romania \\ email: fzoltan@math.ubbcluj.ro
}

Dedicated to the memory of Professor Antal Bege

\begin{abstract}
We establish quantitative estimates for the limit q-Bernstein operator introduced in [3], via the second order Ditzian-Totik modulus of smoothness.
\end{abstract}

\section{Introduction}

The q-Bernstein operators were introduced by Phillips in [8] and they generalize the well-known Bernstein operators. A survey of the obtained results and references concerning q-Bernstein operators can be found in [6]. It is worth mentioning that the first generalization of the Bernstein operators based on q-integers was obtained by Lupaş [4].

Let $q>0$. For each nonnegative integer $k$, the $q$-integers $[k] \equiv[k]_{q}$ and the q-factorials [k]! are defined by

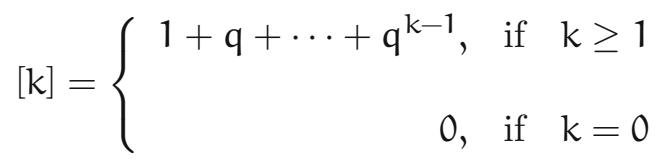

2010 Mathematics Subject Classification: 41A25, 41A36

Key words and phrases: q-Bernstein operators, limit q-Bernstein operator, Ditzian-Totik modulus of smoothness, K-functional 
and

$$
[k] !=\left\{\begin{aligned}
{[1][2] \ldots[k], } & \text { if } k \geq 1 \\
1, & \text { if } k=0 .
\end{aligned}\right.
$$

For integers $0 \leq k \leq n$, the q-binomial coefficients are defined by

$$
\left[\begin{array}{l}
n \\
k
\end{array}\right]=\frac{[n] !}{[k] ![n-k] !}
$$

The $\mathrm{q}$-Bernstein operators $\mathrm{B}_{\mathrm{n}, \mathrm{q}}: \mathrm{C}[0,1] \rightarrow \mathrm{C}[0,1]$ are given by

$$
\left(B_{n, q} f\right)(x) \equiv B_{n, q}(f, x)=\sum_{k=0}^{n} f\left(\frac{[k]}{[n]}\right) p_{n, k}(q, x),
$$

where $\mathrm{n}=1,2, \ldots, 0<\mathrm{q} \leq 1, x \in[0,1]$ and

$$
p_{n, k}(q, x)=\left[\begin{array}{l}
n \\
k
\end{array}\right] x^{k}(1-x)(1-x q) \ldots\left(1-x q^{n-k-1}\right)
$$

for $\mathrm{k}=0,1, \ldots, \mathrm{n}$ (an empty product is taken to be equal 1 ). For $\mathrm{q}=1$ we recover the Bernstein operators. In [8], it is proved the uniform convergence of $B_{n, q_{n}} f$ to $f$ on $[0,1]$, as $n \rightarrow \infty$, when $q=q_{n} \in(0,1)$ and $q_{n} \rightarrow 1$ as $n \rightarrow \infty$. Let $q \in(0,1)$ and $f \in C[0,1]$ be given. Il'inskii and Ostrovska proved in [3] that the sequence $\left\{B_{n, q}(f, x)\right\}$ converges to $B_{\infty, q}(f, x)$ as $n \rightarrow \infty$, uniformly for $x \in[0,1]$, where the limit $\mathrm{q}$-Bernstein operator $\mathrm{B}_{\infty, \mathrm{q}}: \mathrm{C}[0,1] \rightarrow \mathrm{C}[0,1]$ is defined by

$$
\begin{array}{r}
\left(B_{\infty, q} f\right)(x) \equiv B_{\infty, q}(f, x) \\
=\left\{\begin{array}{rr}
\sum_{k=0}^{\infty} f\left(1-q^{k}\right) \frac{x^{k}}{(1-q)^{k}[k] !} \prod_{s=0}^{\infty}\left(1-x q^{s}\right), & \text { if } 0 \leq x<1 \\
f(1), & \text { if } x=1 .
\end{array}\right.
\end{array}
$$

The approximation of continuous functions $f$ by $B_{\infty, q} f$ as $q \nearrow 1$, has been investigated by Videnskii in [9]. We cite the following result of Videnskii. If $0<\mathrm{q}<1, x \in[0,1]$ and $\mathrm{f} \in \mathrm{C}[0,1]$, then

$$
\left|B_{\infty, q}(f, x)-f(x)\right| \leq 2 \omega\left(f, \frac{1}{2} \sqrt{1-q}\right),
$$


where $\omega(f, \delta)=\sup \{|f(x)-f(y)|: x, y \in[0,1],|x-y| \leq \delta\}$ is the usual modulus of continuity of $f$. For the second modulus of smoothness of $f$, defined by

$$
\omega^{2}(f, \delta)=\sup _{0<h \leq \delta} \sup _{x \in[0,1-2 h]}|f(x+2 h)-2 f(x+h)+f(x)|,
$$

Wang obtained the following estimate (see [10] and [11]):

$$
\left|B_{n, q}(f, x)-B_{\infty, q}(f, x)\right| \leq C \omega^{2}\left(f, \sqrt{q^{n}}\right),
$$

where $n=1,2, \ldots, x \in[0,1], 0<q<1$ and $f \in C[0,1]$. Here we mention that $\mathrm{C}>0$ is a constant independent of $n, x$ and $q$, which can be different at each occurrence.

The goal of the paper is to establish quantitative results for the rate of convergence of (2), obtaining similar estimates to (3) and (4). In our estimates we shall use the second order Ditzian-Totik modulus of smoothness of $f$, defined by

$$
\omega_{\varphi}^{2}(f, \delta)=\sup _{0<h \leq \delta} \sup _{x \pm h \varphi(x) \in[0,1]}|f(x+h \varphi(x))-2 f(x)+f(x-h \varphi(x))|,
$$

where $\varphi(x)=\sqrt{\chi(1-\chi)}, x \in[0,1]$ (for details see [1]). Further, we consider the following K-functional:

$$
K_{2, \varphi}(f, \delta)=\inf _{g \in W^{2}(\varphi)}\left\{\|f-g\|+\delta\left\|\varphi^{2} g^{\prime \prime}\right\|\right\},
$$

where $\|\cdot\|$ denotes the uniform norm on $C[0,1]$ and $W^{2}(\varphi)=\left\{g \in C[0,1]: g^{\prime} \in\right.$ $\left.A C_{\text {loc }}[0,1], \varphi^{2} \mathrm{~g}^{\prime \prime} \in \mathrm{C}[0,1]\right\} ; \mathrm{g}^{\prime} \in \mathrm{AC}_{\text {loc }}[0,1]$ means that $\mathrm{g}$ is differentiable such that $g^{\prime}$ is absolutely continuous on every interval $[a, b] \subset[0,1]$. It is known (see $[1,(2.1 .4)])$ that $\omega_{\varphi}^{2}(f, \sqrt{\delta})$ and $K_{2, \varphi}(f, \delta)$ are equivalent, i.e. there exists C $>0$ such that

$$
\mathrm{C}^{-1} \omega_{\varphi}^{2}(f, \sqrt{\delta}) \leq \mathrm{K}_{2, \varphi}(\mathrm{f}, \delta) \leq \mathrm{C} \omega_{\varphi}^{2}(\mathrm{f}, \sqrt{\delta}) .
$$

\section{Main results}

Theorem 1 There exists $\mathrm{C}>0$ such that

$$
\left\|B_{\infty, q} f-f\right\| \leq C \omega_{\varphi}^{2}(f, \sqrt{1-q})
$$

for all $\mathrm{f} \in \mathrm{C}[0,1]$ and $\mathrm{q} \in(0,1)$. Consequently, $\mathrm{B}_{\infty, \mathrm{q}} \mathrm{f}$ converges uniformly to $\mathrm{f}$ on $[0,1]$ as $\mathrm{q} \nearrow 1$. 
Proof. By $[9,(7.7)-(7.8)]$, we have $\mathrm{B}_{\infty, \mathrm{q}}(1, x)=1$ and $\mathrm{B}_{\infty, \mathrm{q}}(\mathrm{t}, \mathrm{x})=\chi$. For $\mathrm{g} \in \mathrm{W}^{2}(\varphi)$, by Taylor's formula:

$$
g(t)=g(x)+g^{\prime}(x)(t-x)+\int_{x}^{t}(t-u) g^{\prime \prime}(u) d u, \quad t, x \in[0,1],
$$

we get

$$
B_{\infty, q}(g, x)-g(x)=B_{\infty, q}\left(\int_{x}^{t}(t-u) g^{\prime \prime}(u) d u, x\right) .
$$

Using the inequality

$$
\left|\int_{x}^{t}(t-u) g^{\prime \prime}(u) d u\right| \leq(t-x)^{2} \varphi^{-2}(x)\left\|\varphi^{2} g^{\prime \prime}\right\|
$$

(see $\left[1\right.$, Lemma 9.6.1]) and $B_{\infty, q}\left((t-x)^{2}, x\right)=(1-q) \varphi^{2}(x)($ see $[9,(7.12)])$, we find

$$
\begin{aligned}
\left|B_{\infty, q}(g, x)-g(x)\right| & \leq B_{\infty, q}\left(\left|\int_{x}^{t}\right| t-u \| g^{\prime \prime}(u)|d u|, x\right) \\
& \leq B_{\infty, q}\left((t-x)^{2}, x\right) \varphi^{-2}(x)\left\|\varphi^{2} g^{\prime \prime}\right\| \\
& =(1-q)\left\|\varphi^{2} g^{\prime \prime}\right\| .
\end{aligned}
$$

On the other hand, by $(2)$ and $B_{\infty, q}(1, x)=1$, we obtain $\left|B_{\infty, q}(f, x)\right| \leq$ $\|f\| B_{\infty, q}(1, x)=\|f\|$, i.e.

$$
\left\|B_{\infty, q} f\right\| \leq\|f\|
$$

for all $f \in C[0,1]$. Now, in view of (7) and (8), we get

$$
\begin{aligned}
\left\|B_{\infty, q} f-f\right\| & \leq\left\|B_{\infty, q} f-B_{\infty, q} g\right\|+\left\|B_{\infty, q} g-g\right\|+\|g-f\| \\
& \leq 2\|f-g\|+(1-q)\left\|\varphi^{2} g^{\prime \prime}\right\| \\
& \leq 2\left\{\|f-g\|+(1-q)\left\|\varphi^{2} g^{\prime \prime}\right\|\right\} .
\end{aligned}
$$

Taking the infimum on the right-hand side over all $\mathrm{g} \in \mathrm{W}^{2}(\varphi)$ and using (5), we get the assertion of our theorem.

Remark 1 The main result of [2] provides an estimate for positive linear operators that preserve linear functions. The result was improved in $[7,(2.138)]$, which implies for the limit q-Bernstein operator that

$$
\left\|B_{\infty, q} f-f\right\| \leq \frac{5}{2} \omega_{\varphi}^{2}(f, \sqrt{1-q}), \quad \text { where } \quad \frac{3}{4} \leq q<1 .
$$


Theorem 2 Let $\mathrm{q} \in(0,1)$ be given. Then there exists $\mathrm{C}>0$ such that

$$
\left\|B_{n, q} f-B_{\infty, q} f\right\| \leq \frac{C}{q(1-q)} \omega_{\varphi}^{2}\left(f, \sqrt{q^{n}}\right)
$$

for all $\mathrm{f} \in \mathrm{C}[0,1]$ and $\mathrm{n}=1,2, \ldots$

Proof. Let $g \in \mathrm{W}^{2}(\varphi)$ and $x \in[0,1]$. Then, by [5, (3.2)], we have

$$
B_{n, q}(g, x)-B_{n+1, q}(g, x)=\sum_{k=1}^{n} a_{n, k}(g) p_{n+1, k}(q, x),
$$

where

$$
\begin{aligned}
a_{n, k}(g)= & \frac{[n+1-k]}{[n+1]} g\left(\frac{[k]}{[n]}\right)+q^{n+1-k} \frac{[k]}{[n+1]} g\left(\frac{[k-1]}{[n]}\right) \\
& -g\left(\frac{[k]}{[n+1]}\right) .
\end{aligned}
$$

By Taylor's formula, we find

$$
\begin{aligned}
g\left(\frac{[k]}{[n]}\right)= & g\left(\frac{[k]}{[n+1]}\right)+\left(\frac{[k]}{[n]}-\frac{[k]}{[n+1]}\right) g^{\prime}\left(\frac{[k]}{[n+1]}\right) \\
& +\int_{[k] /[n+1]}^{[k] /[n]}\left(\frac{[k]}{[n]}-u\right) g^{\prime \prime}(u) d u
\end{aligned}
$$

and

$$
\begin{aligned}
g\left(\frac{[k-1]}{[n]}\right)= & g\left(\frac{[k]}{[n+1]}\right)+\left(\frac{[k-1]}{[n]}-\frac{[k]}{[n+1]}\right) g^{\prime}\left(\frac{[k]}{[n+1]}\right) \\
& +\int_{[k] /[n+1]}^{[k-1] /[n]}\left(\frac{[k-1]}{[n]}-u\right) g^{\prime \prime}(u) d u,
\end{aligned}
$$

respectively. Hence, by (10),

$$
\begin{aligned}
a_{n, k}(g)= & \frac{[n+1-k]}{[n+1]} g\left(\frac{[k]}{[n]}\right)+q^{n+1-k} \frac{[k]}{[n+1]} g\left(\frac{[k-1]}{[n]}\right) \\
& -\frac{[n+1-k]+q^{n+1-k}[k]}{[n+1]} g\left(\frac{[k]}{[n+1]}\right) \\
= & \frac{[n+1-k]}{[n+1]}\left(\frac{[k]}{[n]}-\frac{[k]}{[n+1]}\right) g^{\prime}\left(\frac{[k]}{[n+1]}\right)
\end{aligned}
$$




$$
\begin{aligned}
& +\frac{[n+1-k]}{[n+1]} \int_{[k] /[n+1]}^{[k] /[n]}\left(\frac{[k]}{[n]}-u\right) g^{\prime \prime}(u) d u \\
& +\frac{q^{n+1-k}[k]}{[n+1]}\left(\frac{[k-1]}{[n]}-\frac{[k]}{[n+1]}\right) g^{\prime}\left(\frac{[k]}{[n+1]}\right) \\
& +\frac{q^{n+1-k}[k]}{[n+1]} \int_{[k] /[n+1]}^{[k-1] /[n]}\left(\frac{[k-1]}{[n]}-u\right) g^{\prime \prime}(u) d u \\
& =\frac{[n+1-k]}{[n+1]} \int_{[k] /[n+1]}^{[k] /[n]}\left(\frac{[k]}{[n]}-u\right) g^{\prime \prime}(u) d u \\
& +\frac{q^{n+1-k}[k]}{[n+1]} \int_{[k] /[n+1]}^{[k-1] /[n]}\left(\frac{[k-1]}{[n]}-u\right) g^{\prime \prime}(u) d u,
\end{aligned}
$$

because

$$
\begin{aligned}
\frac{[n+1-k]}{[n+1]}\left(\frac{[k]}{[n]}-\frac{[k]}{[n+1]}\right)+\frac{q^{n+1-k}[k]}{[n+1]}\left(\frac{[k-1]}{[n]}-\frac{[k]}{[n+1]}\right) \\
=\frac{[k]}{[n][n+1]^{2}}\{[n+1-k]([n+1]-[n]) \\
\left.\quad+q^{n+1-k}([k-1][n+1]-[k][n])\right\} \\
=\frac{[k]}{[n][n+1]^{2}}\left\{[n+1-k] q^{n}+q^{n+1-k}\left(-q^{k-1}[n+1-k]\right)\right\} \\
=0 .
\end{aligned}
$$

In view of (6) and (11), we have

$$
\begin{aligned}
\left|a_{n, k}(g)\right| \leq & \frac{[n+1-k]}{[n+1]}\left(\frac{[k]}{[n]}-\frac{[k]}{[n+1]}\right)^{2} \varphi^{-2}\left(\frac{[k]}{[n+1]}\right)\left\|\varphi^{2} g^{\prime \prime}\right\| \\
& +\frac{q^{n+1-k}[k]}{[n+1]}\left(\frac{[k-1]}{[n]}-\frac{[k]}{[n+1]}\right)^{2} \varphi^{-2}\left(\frac{[k]}{[n+1]}\right)\left\|\varphi^{2} g^{\prime \prime}\right\| \\
= & \left\{\frac{[n+1-k][k]([n+1]-[n])^{2}}{[n]^{2}[n+1]([n+1]-[k])}\right. \\
& \left.+\frac{q^{n+1-k}([k-1][n+1]-[k][n])^{2}}{[n]^{2}[n+1]([n+1]-[k])}\right\}\left\|\varphi^{2} g^{\prime \prime}\right\| \\
= & \left\{\frac{[n+1-k][k] q^{2 n}}{[n]^{2}[n+1] q^{k}[n+1-k]}\right.
\end{aligned}
$$




$$
\begin{aligned}
& \left.+\frac{q^{n+1-k}\left(-q^{k-1}[n+1-k]\right)^{2}}{[n]^{2}[n+1] q^{k}[n+1-k]}\right\}\left\|\varphi^{2} g^{\prime \prime}\right\| \\
= & \frac{q^{n-1}}{[n]^{2}[n+1]}\left\{q^{n+1-k}[k]+[n+1-k]\right\}\left\|\varphi^{2} g^{\prime \prime}\right\| \\
= & \frac{q^{n-1}}{[n]^{2}}\left\|\varphi^{2} g^{\prime \prime}\right\| \leq q^{n-1}\left\|\varphi^{2} g^{\prime \prime}\right\| .
\end{aligned}
$$

Hence, by (9) and $B_{n+1, q}(1, x)=1$ (see $[9,(2.5)]$ ), we find

$$
\left|B_{n, q}(g, x)-B_{n+1, q}(g, x)\right| \leq q^{n-1}\left\|\varphi^{2} g^{\prime \prime}\right\|
$$

for all $x \in[0,1]$. This implies that

$$
\begin{aligned}
\| B_{n, q} g- & B_{n+p, q} g \| \\
\leq & \left\|B_{n, q} g-B_{n+1, q} g\right\|+\left\|B_{n+1, q} g-B_{n+2, q} g\right\| \\
& +\cdots+\left\|B_{n+p-1, q} g-B_{n+p, q} g\right\| \\
\leq & \left(q^{n-1}+q^{n}+\cdots+q^{n+p-2}\right)\left\|\varphi^{2} g^{\prime \prime}\right\| \\
\leq & \frac{q^{n-1}}{1-q}\left\|\varphi^{2} g^{\prime \prime}\right\|
\end{aligned}
$$

for $n, p=1,2, \ldots$ In conclusion $\left\{B_{n, q} g\right\}$ is a Cauchy-sequence in $C[0,1]$, so $\left\{B_{n, q} g\right\}$ converges to $B_{\infty, q} g$ as $n \rightarrow \infty$ (see also [3]). Now let $p \rightarrow \infty$ in (12). Then we obtain

$$
\left\|B_{n, q} g-B_{\infty, q} g\right\| \leq \frac{q^{n}}{q(1-q)}\left\|\varphi^{2} g^{\prime \prime}\right\| .
$$

Further, by (1) and $B_{n, q}(1, x)=1$ (see $[9,(2.5)]$ ), we obtain $\left|B_{n, q}(f, x)\right| \leq$ $\|f\| B_{n, q}(1, x)=\|f\|$, i.e.

$$
\left\|B_{n, q} f\right\| \leq\|f\|
$$

for all $f \in C[0,1]$. Then (14), (8) and (13) imply that

$$
\begin{aligned}
\left\|B_{n, q} f-B_{\infty, q} f\right\| \leq & \left\|B_{n, q} f-B_{n, q} g\right\|+\left\|B_{n, q} g-B_{\infty, q} g\right\| \\
& +\left\|B_{\infty, q} g-B_{\infty, q} f\right\| \\
\leq & 2\|f-g\|+\frac{q^{n}}{q(1-q)}\left\|\varphi^{2} g^{\prime \prime}\right\| \\
\leq & \frac{2}{q(1-q)}\left\{\|f-g\|+q^{n}\left\|\varphi^{2} g^{\prime \prime}\right\|\right\} .
\end{aligned}
$$

Taking the infimum on the right-hand side over all $g \in W^{2}(\varphi)$ and using (5), we get the assertion of our theorem. 
Remark 2 Because $\omega_{\varphi}^{2}(f, \delta) \leq \mathrm{C} \omega^{2}(f, \delta) \leq 2 \mathrm{C} \omega(f, \delta)$ (for details see [1]), we obtain, in view of Theorem 1 and Theorem 2, the following weaker estimates:

$$
\left|B_{\infty, q}(f, x)-f(x)\right| \leq C \omega(f, \sqrt{1-q})
$$

and

$$
\left|B_{\infty, q}(f, x)-B_{n, q}(f, x)\right| \leq \frac{C}{q(1-q)} \omega^{2}\left(f, \sqrt{q^{n}}\right) .
$$

\section{References}

[1] Z. Ditzian, V. Totik, Moduli of smoothness, Springer, Berlin, 1987.

[2] I. Gavrea, H. Gonska, R. Păltănea, G. Tachev, General estimates for the Ditzian-Totik modulus, East J. Approx., 9 (2) (2003), 175-194.

[3] A. Il'inskii, S. Ostrovska, Convergence of generalized Bernstein polynomials, J. Approx. Theory, 116 (2002), 100-112.

[4] A. Lupaş, A q-analogue of the Bernstein operator, Babeş-Bolyai University, Seminar on Numerical and Statistical Calculus, 9 (1987), 85-92.

[5] H. Oruç, G. M. Phillips, A generalization of the Bernstein polynomials, Proc. Edinb. Math. Soc., 42 (1999), 403-413.

[6] S. Ostrovska, The first decade of the q-Bernstein polynomials: results and perspectives, J. Math. Anal. Approx. Theory, 2 (1) (2007), 35-51.

[7] R. Păltănea, Approximation theory using positive linear operators, Birkhäuser, Boston, 2004.

[8] G. M. Phillips, Bernstein polynomials based on the q-integers, Ann. Numer. Math., 4 (1997), 511-518.

[9] V. S. Videnskii, On some classes of q-parametric positive operators, $O p$ erator Theory: Advances and Applications, 158 (2005), 213-222.

[10] H. Wang, Korovkin-type theorem and applications, J. Approx. Theory, 132 (2005), 258-264.

[11] H. Wang, F. Meng, The rate of convergence of q-Bernstein polynomials for $0<\mathrm{q}<1$, J. Approx. Theory, 136 (2005), 151-158. 